\title{
Introduction of new multidrug-resistant Salmonella enterica strains into commercial dairy herds
}

\author{
B. Adhikari, ${ }^{*}$ T. E. Besser,† J. M. Gay, ${ }^{*}$ L. K. Fox, ${ }^{*}$ M. A. Davis,† R. N. Cobbold,† A. C. Berge, ${ }^{*}$ R. McClanahan, ${ }^{\star}$ \\ and D. D. Hancock*1 \\ ${ }^{*}$ Agricultural Animal Health Program, Field Disease Investigation Unit, Department of Veterinary Clinical Sciences, and \\ †Department of Veterinary Microbiology and Pathology, Washington State University, Pullman 99164 \\ ¥School of Veterinary Science, The University of Queensland, Brisbane, Queensland 4072, Australia
}

\section{ABSTRACT}

A longitudinal observational study of 59 dairy herds was conducted in Washington State to estimate the rate of introduction of new multidrug-resistant (MDR) Salmonella enterica strains onto commercial dairy herds. Samples were collected on these herds over 7 visits separated by intervals of 2 to 4 mo over a period of 15 to 21 mo. Samples were cultured for Salmonella spp. and serogroup, serovar, and antimicrobial susceptibility patterns were identified for MDR Salmonella isolates. Fingerprinting generated by pulsed-field gel electrophoresis (PFGE) using $\mathrm{XbaI}$ restriction enzyme digestion generated genotyping profiles for all MDR isolates identified in the study. The rate of new MDR Salmonella strain introduction was 0.9 per herd-year (95\% confidence interval: 0.6-1.4). The rates for the most commonly introduced MDR Salmonella serovars were 0.4 /herd-year for Typhimurium, 1.2/herd-year for Newport, and 0.1/herd-year for Dublin. Thirty-three of 59 herds (56\%) had at least one new MDR Salmonella introduction during the study period. The number of new MDR Salmonella strains acquired by dairy herds ranged from zero to 8 . Thirteen of the 59 herds had a history of clinical salmonellosis. Among these 13 herds, 6 herds acquired new MDR Salmonella strains, although these strains were different than historical clinical strains. These data indicate that acquisition of new MDR Salmonella strains by dairy herds was a common event in participating herds, although the number of strains introduced varied greatly among herds.

Key words: multidrug-resistant Salmonella, dairy farm, incidence rate, introduction

Received June 27, 2008.

Accepted February 24, 2009.

${ }^{1}$ Corresponding author: hancock@wsu.edu

\section{INTRODUCTION}

Salmonella enterica is a common zoonotic pathogen that is one of the leading causes of foodborne bacterial infections in the United States (CDC, 2005). An estimated 1.3 million human clinical salmonellosis cases and more than 500 human deaths associated with Salmonella may occur annually in the United States (Mead et al., 1999). Domestic livestock are considered the main reservoir for foodborne salmonellosis including strains with multidrug resistance (Sanchez et al., 2002).

Zoonotic bacteria demonstrating antimicrobial resistance are an emerging problem worldwide (FAO/WHO, 2007). Transmission of zoonotic MDR Salmonella is of particular concern because of the limited effectiveness of first-line antimicrobials when treating infections, particularly pediatric infections, and because drug-resistant strains may be more likely to cause prolonged or severe illnesses than drug-susceptible strains (Butaye et al., 2006). Publications of Salmonella transmission from animals to humans include meat or milk from cattle (Spika et al., 1987), meat from poultry (McPherson et al., 2006), and infection through direct contact with infected cattle (Bezanson et al., 1983; Fey et al., 2000). Multidrug resistance is a characteristic of many newly emerging strains that are believed to spread mainly by clonal expansion (Hancock et al., 2000; Davis et al., 2002, 2007a; Velge et al., 2005).

Several studies have been published on the epidemiology of Salmonella and risk factors associated with Salmonella transmission between cattle herds. A recent study in the southwestern United States reported a low level of risk of Salmonella transmission from the heifer feedlot back to the dairy herds (Edrington et al., 2008). In previous studies, the transport of infected cattle into cattle herds has been described as the primary route of interherd transmission of Salmonella spp. (Wray et al., 1990; Evans, 1996; Zansky et al., 2002). Salmonella prevalence in preweaned calves has been shown to be lower in herds that are closed (i.e., no cattle are introduced into the herd; Berge et al., 2006). Other studies 
Table 1. Attributes of 59 northwestern US commercial dairy herds enrolled in this study

\begin{tabular}{lrc}
\hline Attribute & Value & Range \\
\hline Median herd size (dairy cattle of all ages), n & 1,800 & $231-17,775$ \\
Median number of lactating cows, n & 750 & $100-7,150$ \\
Median number of dry cows, n & 130 & $0-1,409$ \\
Median number of calves <3 mo on-farm, n & 80 & $0-3,000$ \\
Median number of calves <3 mo off-farm, n & 0 & $0-685$ \\
Median number of heifers from 3 to 20 mo on-farm, n & 230 & $0-3,500$ \\
Median number of heifers from 3 to 20 mo off-farm, n & 40 & $0-5,600$ \\
Median number of heifers from 20 mo to calving off-farm, n & 100 & $0-700$ \\
Farms that introduced new animals during the study, $\%$ & 53 & - \\
Median number of cattle introduced, n & 2 & $0-1,340$ \\
Farms that raised any cattle off-farm, \% & 47 & - \\
\hline
\end{tabular}

${ }^{1}$ New animal introduction into the farm was defined as new animal purchase or returning cattle from a contract-heifer raising operation or owned cattle raised offsite where cattle mingled with cattle from other sources during the study period.

have shown that Salmonella transmission can occur via movements of people, equipment, fomites, wild animals, and through environmental contamination (Langvad et al., 2006; Nielsen et al., 2007a). Direct cattle-to-cattle contact from other herds can also result in the introduction of Salmonella spp. into dairy farms (van Schaik et al., 2002). The rate of cattle introduction from testpositive herds has been shown to be associated with recipient herds changing from Salmonella-negative status to positive (Nielsen et al., 2007b). Another potential source of Salmonella transmission includes livestock feed (Anderson et al., 2001; Davis et al., 2003).

Relatively few studies have been performed to examine the rate at which Salmonella spp. are introduced onto commercial dairy herds. In the United Kingdom, a previous study reported an incidence rate of 0.43 cases of salmonellosis per farm-year at risk for any serovar of Salmonella enterica (Davison et al., 2006). No data are currently available on the incidence of MDR Salmonella strain introduction into dairy herds in North America. Such information is useful in undertaking risk assessments for human transmission (FAO/WHO, 2007). Therefore, the purpose of this study was to estimate the rate at which MDR Salmonella strains were introduced into commercial dairy herds in the northwestern United States.

\section{MATERIALS AND METHODS}

\section{Study Herds}

Fifty-nine commercial dairy herds ranging in size from 231 to 17,775 head (cattle of all ages) in Washington State were sampled during the period from August 2005 to December 2007. According to the Dairy Farmers of Washington State (http://www.havemilk.com; 2007), there were 496 dairy herds and 238,000 milk cows in Washington State. Dairy herds were selected across the 3 major dairy areas (western, central, and south-central) of Washington State, proportional to the total dairy herds in that region. Sixty-six herds were randomly selected alphabetically from the list of herds, and contact was made through telephone and by herd veterinarians to explain the project. Sixty-six dairy herds were enrolled, 6 declined participation, and 1 farm went out of business during the study, leaving us with 59 herds for study. In Washington State, herd veterinarians regularly submit fecal samples to Washington State Disease Diagnostic Laboratory for suspected cases of salmonellosis. Management-related information was collected via a standardized questionnaire and by on-site inspection (Table 1).

\section{Sampling Plan}

Each farm was visited 7 times to collect samples at intervals of 2 to 4 mo over a period of 15 to 21 mo from August 2005 to December 2007. In total, 7,009 pooled fecal samples were collected from the 59 dairy herds (Table 2). Each fecal pool consisted of approximately $5 \mathrm{~g}$ of feces collected from 10 individual, undisturbed fresh fecal pats from the pen floor using sterile tongue depressors. An average of 16 fecal pools was collected per farm per visit from different groups of cattle in the farm. The number of pools collected from each group was proportional to the group size. Pooled fecal samples were obtained from hutch calves (1), heifers (1-2), lactating cows (3-9), dry cows (2-3), close-up dry cows $(2-3)$, and cows in the maternity/hospital area (1-2). Certain groups of cattle (calves, lactating cows, maternity/hospital cows) were selectively sampled to increase sensitivity, as Salmonella prevalence is reported to be greater in these groups than in the general herd (Hancock, 1996; Warnick et al., 2003). This sampling method was estimated to provide at least a $95 \%$ probability of detecting a positive herd if the within-herd prevalence 
Table 2. Number of farm visits, samples, and sample sources per farm visit from August 2005 through December 2007

\begin{tabular}{lcccccc}
\hline & & \multicolumn{5}{c}{ Samples, $\mathrm{n}$} \\
\cline { 3 - 6 } $\begin{array}{l}\text { Farm visit } \\
\text { number }^{1}\end{array}$ & $\begin{array}{c}\text { Total farm } \\
\text { visits, } \mathrm{n}\end{array}$ & Pooled feces $^{2}$ & Pooled feed $^{3}$ & Milk filter $^{4}$ & Slurry & Total \\
\hline 1 & 59 & 1,274 & 664 & 50 & 82 & 2,129 \\
2 & 59 & 955 & 644 & 52 & 71 & 1,781 \\
3 & 59 & 950 & 631 & 53 & 50 & 1,743 \\
4 & 59 & 976 & 654 & 51 & 53 & 1,793 \\
5 & 59 & 920 & 690 & 51 & 49 & 1,769 \\
6 & 59 & 980 & 667 & 33 & 56 & 1,795 \\
7 & 59 & 954 & 632 & 50 & 53 & 1,748 \\
Total & 413 & 7,009 & 4,582 & 340 & 414 & 12,758 \\
\hline
\end{tabular}

${ }^{1}$ The interval between visits ranged from 2 to 4 mo.

${ }^{2}$ Pooled fecal samples per farm ranged from 15 to 21 .

${ }^{3}$ Feed collected from 5 different areas of particular feed type in each feed storage area.

${ }^{4}$ Some farms did not have a milk filter at time of visit.

was $8 \%$ or more (Jordan, 2005). Initial sample size estimates were based on recovery of Salmonella from a herd. The samples were thereafter targeted to high-risk commodities and animals to recover as many different types of Salmonella as could be present.

Samples of all available component feed types were collected from respective farms and from 2 feed mills that supplied feeds to most dairy herds enrolled in the study. Each pooled feed sample consisted of approximately $10 \mathrm{~g}$ of feed collected from 5 different areas of a particular feed type in each feed storage unit. On average, 10 feed pools were collected from each farm per visit. Feed samples were collected using sterile gloves after removing 2.5 to $5 \mathrm{~cm}$ of the top surface layer of feed. Samples were transported to the laboratory at 4 to $8^{\circ} \mathrm{C}$ and were processed within $24 \mathrm{~h}$ of collection.

Other samples included slurry and milk filters. Using sterile gloves, up to 2 slurry samples of $50 \mathrm{~mL}$ from the slurry pond, and up to 2 milk filters from the trash bin were collected from each dairy farm.

\section{Salmonella Culture and Identification}

For isolation of Salmonella spp., $12.5 \mathrm{~g}$ of each fecal sample was enriched in $112.5 \mathrm{~mL}$ of tetrathionate broth (TB, Hardy Diagnostics, Santa Maria, CA) and $112.5 \mathrm{~mL}$ of Rappaport-Vassiliadis broth (R10; Hardy Diagnostics) and incubated for $24 \mathrm{~h}$ at $37^{\circ} \mathrm{C}$. Enriched samples were plated onto xylose lactose tergitol-4 agar (XLT-4, Hardy Diagnostics) and MacConkey (Mac, Hardy Diagnostics) agar plates supplemented with ampicillin $(256 \mu \mathrm{g} / \mathrm{mL})$, chloramphenicol $(8 \mu \mathrm{g} / \mathrm{mL})$, and streptomycin $(32 \mu \mathrm{g} / \mathrm{mL}$, Sigma-Aldrich, Dallas, TX) and incubated for $24 \mathrm{~h}$ at $37^{\circ} \mathrm{C}$. Feed $(25 \mathrm{~g})$ or slurry $(25 \mathrm{~mL})$ samples were pre-enriched in $225 \mathrm{~mL}$ of buffered peptone water (BPW, Hardy Diagnostics) and incubated for $24 \mathrm{~h}$ at $37^{\circ} \mathrm{C}$ before performing paral- lel enrichments by transferring $1 \mathrm{~mL}$ of BPW to $9 \mathrm{~mL}$ of TB and $100 \mu \mathrm{L}$ of BPW to $10 \mathrm{~mL}$ of R10, incubating for $48 \mathrm{~h}$ at $42^{\circ} \mathrm{C}$, and cross-plating onto XLT-4 and Mac media. The fecal Salmonella isolation protocol was used for isolation of Salmonella from milk filters. For confirmation of suspected Salmonella, 3 colonies from XLT-4 and Mac were inoculated onto lysine iron agar slants and incubated for $24 \mathrm{~h}$ at $37^{\circ} \mathrm{C}$. Salmonella suspects from lysine iron agar were transferred to triple sugar iron agar slants and subsequently to urea agar slants incubated 18 to $24 \mathrm{~h}$ at $37^{\circ} \mathrm{C}$. One isolate per sample was further screened with agglutination tests for serogroup identification using commercial polyvalent A-I and Vi antisera (Difco Laboratory, Detroit, $\mathrm{MI}$ ). Isolates were then banked in brain-heart infusion broth containing 25 to $30 \%$ buffered glycerol and stored at $80^{\circ} \mathrm{C}$. One isolate per sample (except when different serogroups were detected from the same sample either on single or 2 different culture media) was selected for further characterization with antimicrobial susceptibility testing.

\section{Antimicrobial Susceptibility Testing}

Antimicrobial susceptibility testing was performed using the Kirby-Bauer disk diffusion method (Bauer et al., 1966). Each isolate was classified as resistant or susceptible to each antimicrobial tested by the threshold zone size for resistance as recommended by Clinical and Laboratory Standards Institute (CLSI) guidelines (NCCLS, 2003a,b) using the following antimicrobial drugs (BD Diagnostics, Sparks, MD): ampicillin $(10 \mu \mathrm{g})$, ceftazidime $(30 \mu \mathrm{g})$, chloramphenicol $(30 \mu \mathrm{g})$, gentamicin $(10 \mu \mathrm{g})$, amoxicillin-clavulanic acid $(20 / 10 \mu \mathrm{g})$, kanamycin $(30 \mu \mathrm{g})$, streptomycin $(10 \mu \mathrm{g})$, tetracycline $(30 \mu \mathrm{g})$, triple-sulfa (a combination of sulfadiazine, sulfamethazine, and sulfamerazine; $250 \mu \mathrm{g}$ ), 
and trimethoprim-sulfamethoxazole $(1.25 / 23.75 \mu \mathrm{g})$. Isolates showing antimicrobial resistance to 2 or more antimicrobials belonging to distinct classes of antimicrobial agents were designated as MDR isolates. A total of 385 MDR Salmonella isolates (one isolate per sample per visit except if more than one serogroups detected in a sample) from the current study and 26 historical clinical MDR Salmonella isolates collected before the study were serotyped at the National Veterinary Service Laboratory (Ames, IA). A 3-tube most-probable-number (MPN) method was used to determine the level of Salmonella contamination of positive feeds (Blodgett, 2006).

\section{Pulsed-Field Gel Electrophoresis}

All MDR Salmonella isolates from fecal samples were analyzed using pulsed-field gel electrophoresis (PFGE) following $\mathrm{XbaI}$ restriction digestion based on the PulseNet protocol for Salmonella and using Salmonella Braenderup H9812 as a size standard (Ribot et al., 2002). The PFGE images were analyzed using BioNumerics software (v. 3.5, Applied Maths, SintMartens-Latem, Belgium). Independent strains were defined by PFGE profiles that differed by at least 2 bands with a position tolerance of $1.5 \%$.

\section{Data Analysis}

For the purpose of this study, a strain found on the farm was considered a newly introduced strain if the strain was isolated during the second through seventh sampling visits and had not been previously observed on that farm in the first sampling visit and in diagnostic samples from suspected clinical cases for at least 3 or more years before the commencement of the study.

Introduction of new strains was not based on serotypes, but on PFGE profiles, where we have designated a new strain for isolates from farm visits 2 to 7 that had a PFGE banding pattern different (based on at least 2 PFGE band differences) from historical and baseline isolates (visit 1). Herd classification at the start of the study (visit 1) was based on isolation of Salmonella from any source (pooled feces, feed, milk filters, or slurry). New introductions of Salmonella spp. were based purely on isolation of Salmonella from pooled feces specimens.

Incidence rates of introduction of new MDR Salmonella strain into the herds were calculated using Excel 2003 (Microsoft, Redmond, WA) and SAS version 9.1 (SAS Institute Inc., Cary, NC). Incidence rates for events, including $95 \%$ confidence intervals (CI), were calculated using a Poisson distribution and expressed as new strains per herd-year at risk. Overall MDR in- cidence rate was calculated as the number of specific Salmonella serovar-negative herds at commencement of sampling that become positive, divided by the total observation time at risk. The incidence rates were calculated by using either the serovar-specific status of the herds or their overall new MDR Salmonella spp. status. A herd was considered at risk for new MDR Salmonella strains until the herd was positive for that respective MDR Salmonella strain.

\section{RESULTS}

\section{Description of New Strain Introduction}

Sixty-six dairy herds were enrolled, 6 declined participation, and 1 farm went out of business during study period. The study thereby included 59 herds containing 173,253 cattle with farm size and geographical distributions representative of Washington State. A total of 7,009 fecal and 4,582 feed pool samples were collected in these herds. The median total herd size in terms of dairy cattle of all ages and of lactating cows only were 1,800 (range 231-17,775) and 750 (range 100-7,150), respectively (Table 1). Fifty-three percent of the herds introduced new animals during the study period.

Historical information on clinical Salmonella infection from participating herds (Table 3 ) indicated that 13 of 59 herds were diagnosed with clinical salmonellosis before the study, based on Salmonella-positive clinical sample submissions to Washington State Disease Diagnostic Laboratory. A total of 26 MDR Salmonella enterica isolates were detected in historical clinical submission samples. Among the MDR strains, antimicrobial resistance was seen in all 26 isolates to ampicillin; followed by 25 isolates to triple-sulfa, streptomycin, and tetracycline; 16 isolates to chloramphenicol; 14 isolates to ceftazidime; 13 isolates to kanamycin; 4 isolates to trimethoprim-sulfamethoxazole; and 2 isolates to gentamicin. All MDR isolates were resistant to at least 5 antimicrobials, whereas $5(19 \%)$ were resistant to 7 or more antimicrobials. Among the historic clinical MDR strains, $S$. Typhimurium was most common serovar (12/26) followed by $S$. Newport $(9 / 26)$. None of the historical MDR Salmonella strains were isolated in the 7 farm visits. Among 13 herds positive for history of clinical salmonellosis before the study, 5 were positive for MDR Salmonella at the first farm visit, and 8 acquired new strains at farm visit 2 or later.

A total of 70 new introductions of MDR Salmonella strains were observed in 33 dairy herds (Table 4). The most common serovars identified among the new strains were Typhimurium (14 introductions), Newport (10), Dublin (8), Montevideo (3), and Uganda (2). Other serovars included Ohio, Oranienburg, Hadar, Infantis, 
Table 3. Characteristics of Salmonella spp. isolated at farm visit 1 and records of historical clinical isolates from participating dairy herds in Washington State

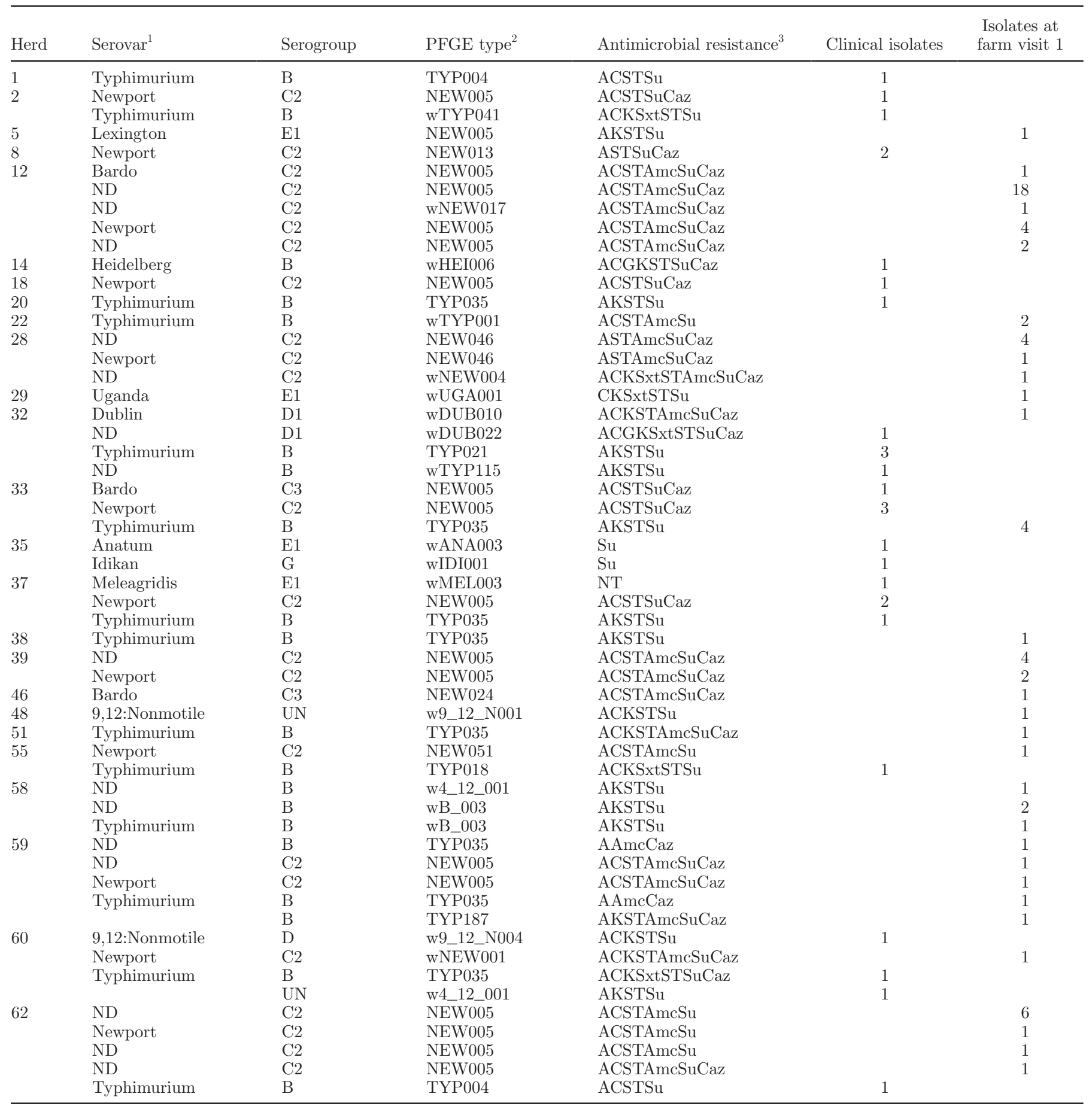

${ }^{1} \mathrm{ND}=$ serotyping was not performed as the herds associated with these isolates never acquired new multidrug-resistant Salmonella strains with corresponding pulsed-field gel electrophoresis (PFGE) types, and research constraints.

${ }^{2}$ PFGE type as designated by Washington Department of Public Health with the exception of types starting with the letter w, which indicates a new PFGE type designated at Washington State University.

${ }^{3} \mathrm{~A}=$ ampicillin; $\mathrm{C}=$ chloramphenicol; $\mathrm{G}=$ gentamicin; $\mathrm{K}=$ kanamycin; $\mathrm{S}=$ streptomycin; $\mathrm{Su}=$ triple sulfa; $\mathrm{T}=$ tetracycline; Caz = ceftazidime; Amc = Amoxicillin-clavulanic acid; Sxt = trimethoprim-sulfamethoxazole; $\mathrm{NT}=$ not tested; UN $=$ unknown. 
Meleagridis, and nonserotypeable isolates. New strains T5, D1, I1, and N1 were acquired by $13(22 \%), 3(5 \%)$, $3(5 \%)$, and $2(3 \%)$ herds, respectively (Table 4$)$. Fiftyeight $(83 \%)$ strains were resistant to 5 or more antimicrobials tested including ceftazidime, a third-generation cephalosporin.

Twenty-six (44\%) herds did not acquire any new introductions of MDR Salmonella, whereas 33 (56\%) herds acquired at least one new introduction of MDR Salmonella during the study period. The number of new introductions of MDR Salmonella on these herds varied from zero to 8 (Figure 1). Eighteen herds acquired 1 new MDR Salmonella strain, 12 herds acquired 2 or 3 new MDR Salmonella strains, and 1 and 2 herds acquired 6 and 8 new introductions, respectively. The incidence rate of introduction of MDR Salmonella strains in Washington State commercial dairy herds was 0.9 per herd-year (95\% CI: 0.6-1.4). The rates of introduction for the most commonly introduced MDR Salmonella serovars were $0.4 /$ herd-year for Typhimurium, 1.2/ herd-year for Newport, and 0.1/herd-year for Dublin.

Table 5 summarizes the temporal and geographical distributions of newly introduced MDR Salmonella serotypes isolated from the study herds. Of the 59 herds, new strains of serovar Typhimurium were acquired by $27(39 \%)$ herds followed by Newport (17\%), Dublin (16\%), Infantis, Montevideo, and Uganda (4\%). Newly introduced strains of serovar I 4,12:nonmotile, Infantis and Newport were isolated from herds in 4 counties of Washington State. Strains of serovars Typhimurium and Oranienburg were isolated from herds in 3 and 2 counties, respectively.

\section{MDR Salmonella Contamination in Feed}

A total of 4,582 pooled feed samples from the 59 herds and 665 feed samples from 2 feed mills were collected over the 7 sampling periods. Of those, $3.5 \%$ (158) of feeds were positive for any Salmonella spp. Of the feed source Salmonella spp., 83, 8, and $9 \%$ were pansusceptible, resistant to 1 antimicrobial, and resistant to 2 or more antimicrobials (i.e., MDR), respectively. Overall, the prevalence of pan-susceptible and MDR Salmonella in feeds were 2.5 and $0.5 \%$, respectively. Feeds from 12 (19\%) herds yielded 12 MDR Salmonella during 413 farm visits (Table 6). Of 665 feed mill samples, only 1 sample $(0.2 \%)$ was positive for MDR Salmonella. This MDR feed mill Salmonella Bardo isolate was from mixed grains and it was never isolated in study herds during the sampling period. Multidrugresistant Salmonella Typhimurium were isolated from several feed types on farms, including straw, corn silage, calf grains, and haylage. Multidrug-resistant $S$. Dublin and $S$. Newport were isolated from calf grain, bakery waste, and cannery waste at these farms, respectively. Salmonella Saint-Paul was the only serovar isolated from cottonseed at a single farm. The MDR Salmonella feed isolates exhibited resistance to 2 to 7 antimicrobials. The MDR Salmonella strains recovered from feed were not recovered from cattle fecal samples on the same farm. Quantitative analysis of feeds positive for MDR Salmonella showed that none of the samples were positive for Salmonella in triplicate MPN dilution series using inoculum quantities of $0.1,0.01$, and $0.001 \mathrm{~g}$ after 48-h enrichment in TB. Thus, the computed MPN concentration of MDR Salmonella for all types of feeds was less than $3 \mathrm{cfu} / \mathrm{g}$. These feeds were positive only by the selective enrichment of $25 \mathrm{~g}$ of feed.

\section{DISCUSSION}

The rate of new MDR Salmonella strain introduction in 59 Washington State herds was 0.9 per herd-year. This rate of introduction is higher than 0.43 cases per farm-year at risk reported in England and Wales (Davison et al., 2005). The estimate from that study also included pan-sensitive Salmonella strains, which accounted for a majority of newly introduced strains. Hence, the rate of MDR Salmonella introduction (not computed separately) in the British study must have been at least 4 times lower than the rate in the present study. This difference is possibly because of differences in herd size, feeding, management, and environmental factors between British herds and western US herds. The most common Salmonella serovars found in the present study were Typhimurium, Newport, and Dublin, whereas in the British study, the most common serovars were Dublin, Agama, and Typhimurium (Davison et al., 2005).

Our finding of the most common Salmonella serovars in the order of Typhimurium, Newport, and Dublin is consistent with the emergence, re-emergence, and increasing isolation of these serovars in previous studies in the northwestern US commercial dairy herds (Berge et al., 2004, 2006; Velge et al., 2005; Davis et al., 2007a, b). The MDR Salmonella strain designated T5 in the present study was acquired by 13 herds. This strain belonged to Salmonella Typhimurium PFGE subtype WA-TYP035/187, which had previously been reported to have emerged and disseminated in dairy herds in the Pacific Northwest and has been seen increasingly in human clinical cases (Davis et al., 2007a). Our data reflect emerging serovars for the herds sampled, which in turn is an indicator of emergent serovars for the region. The concept of regional emergence of strains is exemplified across studies by the T5 strain, which was reported in all dairy herds in the Pacific Northwest. The main purpose of the current study was to describe 
Table 4. Characteristics of newly introduced multidrug-resistant Salmonella strains isolated from fecal samples from 33 of 59 dairy herds from August 2005 through December 2007

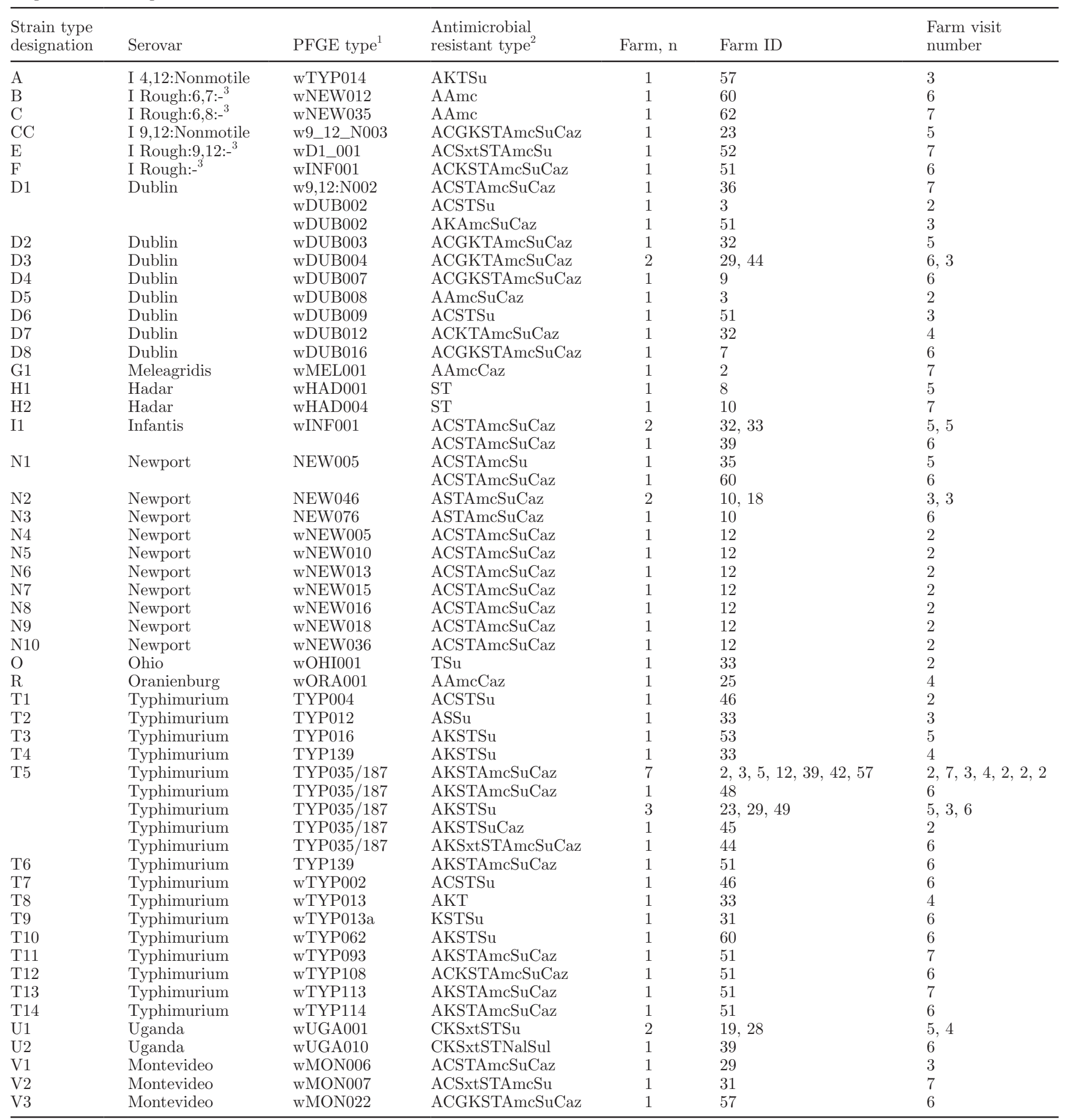

${ }^{1}$ Pulsed-field gel electrophoresis (PFGE) type as designated by Washington Department of Public Health with the exception of types starting with the letter w, which indicates a new PFGE type designated at Washington State University.

${ }^{2} \mathrm{~A}=$ ampicillin; $\mathrm{C}=$ chloramphenicol; $\mathrm{G}=$ gentamicin; $\mathrm{K}=$ kanamycin; $\mathrm{S}=$ streptomycin; $\mathrm{Su}=$ triple sulfa; $\mathrm{T}=$ tetracycline; Caz $=$ ceftazidime; Amc $=$ Amoxicillin-clavulanic acid; Sxt $=$ trimethoprim-sulfamethoxazole; $\mathrm{NT}=$ not tested; $\mathrm{UN}=$ unknown.

${ }^{3}$ Popoff (2001). 
Table 5. Temporal and geographical distribution of newly introduced multidrug-resistant Salmonella enterica serovars isolated from 59 herds in different counties $(\mathrm{n}=7)$ of Washington State

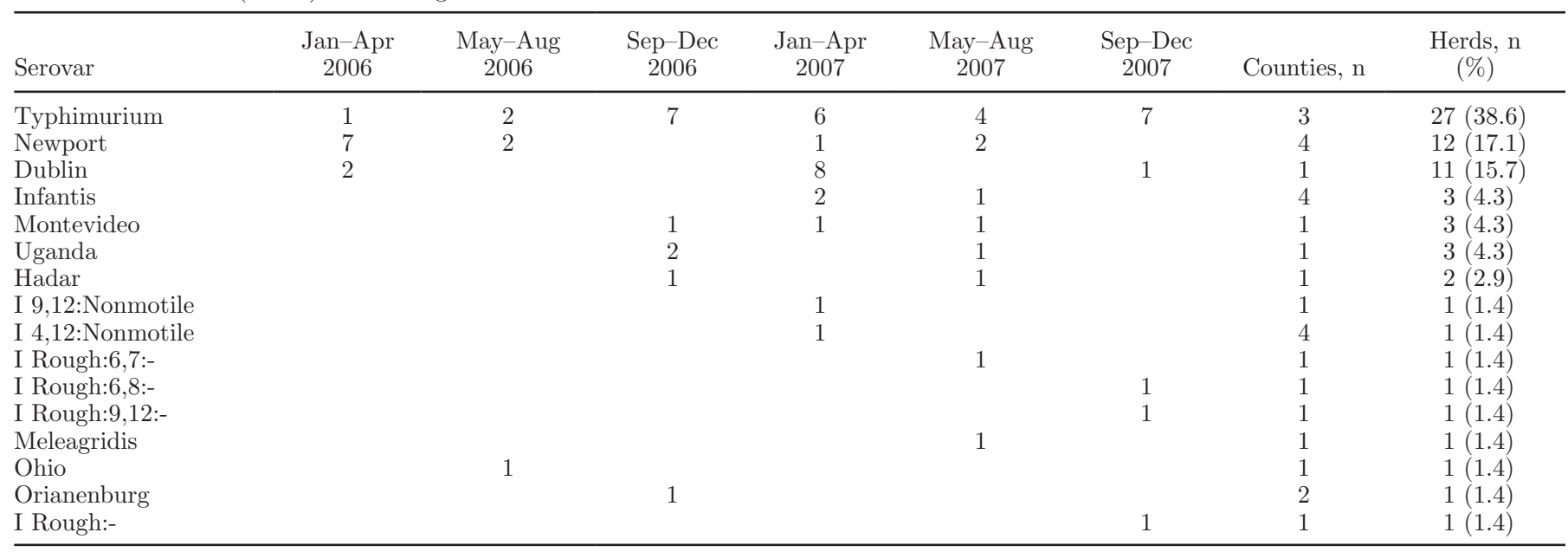

the introduction of new strains not seen before in particular dairy herds.

Although some studies have reported evidence of Salmonella introduction in cattle via feeds (Lindqvist et al., 1999; Davis et al., 2003), several other studies have failed to show direct evidence indicating feed as a potential source of Salmonella infection in cattle (Richardson, 1975; Williams, 1975; Wray and Sojka, 1977). In the current study, most (88\%) of the feed Salmonella isolates were pan-susceptible, and only a very small proportion $(0.5 \%)$ were contaminated with MDR Salmonella. The concentration of MDR Salmonella found in feeds was much lower than that likely to result in an infectious dose in healthy animals (Smith et al., 1979; Spier et al., 1991). Certain factors can reduce the infectious dose in cattle (Chambers and Lysons, 1979; Mattila et al., 1988). Although MDR Salmonella were isolated from feed samples from different herds, we did not find MDR Salmonella feed strains introduced or acquired by dairy herds on the same farm. This does not mean, however, that higher contamination levels do not occur, as has been documented in some studies (Anderson et al., 1997). The rarity and low concentration of MDR Salmonella contamination of feed in the present study do indicate that introduction of MDR Salmonella infections into dairy herds via feed is unlikely to be common.

It was interesting to note that feed samples from feed mills before delivery to the farm were very rarely contaminated with Salmonella. Considering that 665 feed

Table 6. Distribution of multidrug-resistant Salmonella serovars cultured from different feed types from commercial herds $(\mathrm{n}=59)$ in Washington State from August 2005 through December 2007

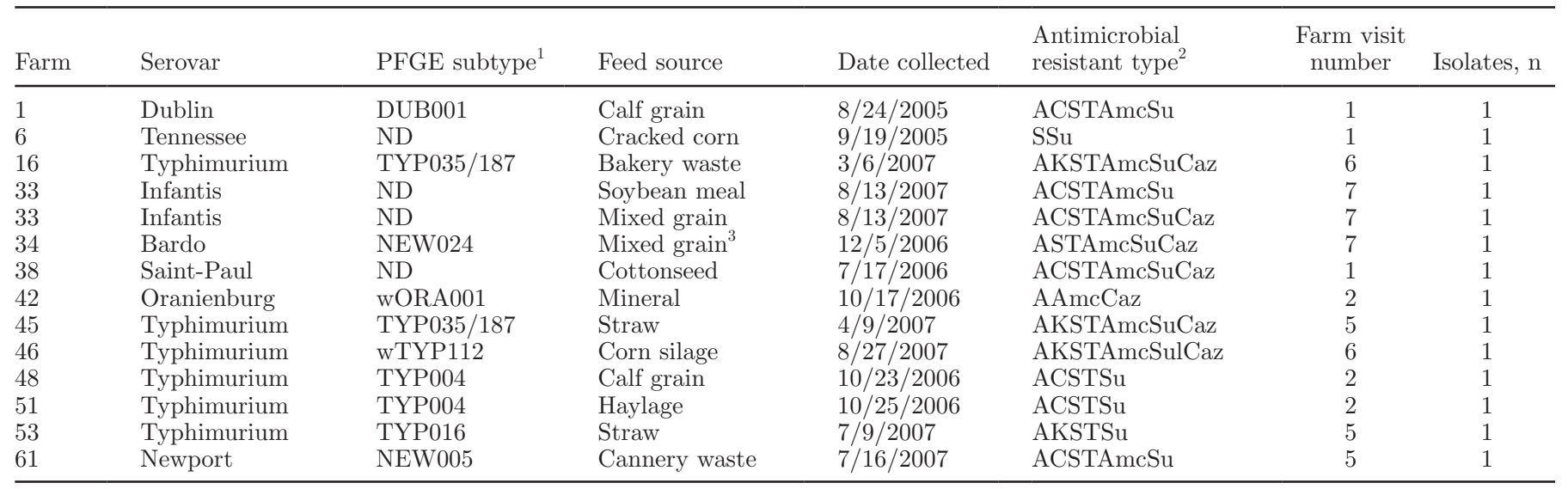

${ }^{1} \mathrm{PFGE}=$ pulsed-field gel electrophoresis; $\mathrm{ND}=$ not done.

${ }^{2}$ See Table 2 for abbreviations.

${ }^{3}$ Feed mill samples before shipment to farms. 


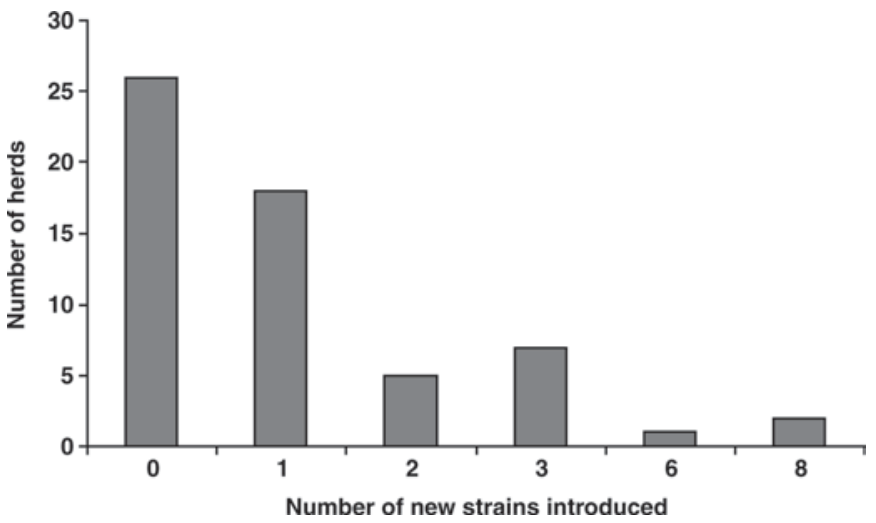

Figure 1. Distribution of 59 dairy herds based on the number of new multidrug-resistant Salmonella strains acquired over 15- to 21-mo sampling periods.

mill samples were analyzed, the power of the study was sufficient to detect Salmonella-positive feed. However, if low-level contamination is present in the feed mill samples and the regrowth of Salmonella only occurs at the farm, then it is possible that our sampling method was not sensitive enough to capture this low-level contamination. Another hypothesis is that the feed was contaminated at the farm. Great care was taken to take a sterile sample below the surface of the pile. There is, however, frequent movement of feed on farms. It may be that most feed contamination at the farm reflects the herd or farm infection rather than the feed being a primary cause of contamination. Although some farm feed was positive for MDR Salmonella, we never isolated feed-associated strains (based on PFGE profiles) from cattle feces on repeated samplings. Because of the low level of contamination and because strains in feed were not found in cattle, we believe that feed is not a major pathway for introduction of new strains in Washington State.

Diagnostic laboratory records indicated that 13 of 59 herds had a history of salmonellosis at some point before the commencement of the study. It is widely believed that Salmonella infections can be subclinical in cattle, and not all clinical cases of salmonellosis would have been submitted for diagnostic testing for Salmonella. Although it is possible that farm selection (based on voluntary participation) could have been influenced by known salmonellosis status, $22 \%$ of herds that had a history of clinical salmonellosis did not acquire any new MDR Salmonella strains in the present study compared with $78 \%$ of herds that had no evidence of previous cases. On the other hand, $70 \%(23 / 33)$ of herds that acquired new MDR Salmonella strains at farm visit 2 or later were negative for MDR Salmonella at farm visit 1 , which indicates that herds that acquired new strains were not likely to have MDR Salmonella at the start of the study.

Culture-dependent isolation of Salmonella spp. is naturally less than $100 \%$ sensitive. However, methods such as pooling of samples, targeted sampling, and sample pre-enrichment were used to improve the recovery of damaged cells and subsequent isolation rates. Initial assessment of Salmonella strains present in the dairy herds was based on more intensive sampling around herds. Certain sample types were targeted (e.g., pooled sampling, milk filters, slurry) that increase the chances of determining herd-wide status. Cattle sampling was divided between different groups of cattle on the farm (e.g., heifers, lactating, dry), as Salmonella and MDRSalmonella prevalence have been demonstrated to vary significantly between these groups (Warnick et al., 2003; Cobbold et al., 2006). It is possible that a pre-existing strain was missed but that the chance of this occurring was minimized through the sampling and microbiological methods employed. Selecting multiple colonies per sample, use of multiple culture media, use of multiple enrichment broths, and samples from targeted cattle groups should maximize the isolation rate, which indirectly reduces the chance of missing a pre-existing strain.

Minor genetic events can change PFGE patterns. Therefore, the possibility exists for misclassification based on PFGE just as with any other typing method. However, PFGE has been found to be of significant value in identifying epidemiologically related isolates in many studies. For the purposes of our study, PFGE was judged to be a reasonable compromise between methods that are more insensitive to detecting different strains (e.g., serotypes, phage types) and those that are more likely to falsely detect new strains because of their high sensitivity to minor genetic changes (e.g., multiple-locus variable-number tandem repeat analysis, amplified fragment length polymorphisms).

In conclusion, our findings suggest that new MDR Salmonella strains are frequently introduced into Washington State commercial dairy herds. Feed probably plays a lesser role in Salmonella transmission to herds than other modes of introduction such as cattle introduction. The herds enrolled in the current study were considered representative of dairy herds in Washington State and may represent similarly managed herds in other regions of the United States. Herds sampled represented a wide range of production practices and herd management types, which in turn are representative of most large-scale and intensive dairying production practices outside the Pacific Northwest. The management factors associated with the rate of Salmonella introduction are described elsewhere (Adhikari et al., 2009). Further studies are needed to determine the 
epidemiology of MDR Salmonella introduction, maintenance, and dissemination at the farm level and to examine whether the patterns of new strain introduction reported in the current study are similar to that in other US dairy herds.

\section{ACKNOWLEDGMENTS}

This project was supported in part by National Institutes of Health, Department of Health and Human Services under the contract number NO1-AI-30055 and by the Agricultural Animal Health Program, College of Veterinary Medicine, Washington State University. The skillful technical assistance of Regina Finger and Katherine Kaya Baker of the Field Disease Investigation Unit (FDIU), College of Veterinary Medicine, Washington State University, is gratefully acknowledged. We also thank Lindsay Tippett, Lisa Jones, and other staff of the FDIU for their technical help.

\section{REFERENCES}

Adhikari, B., T. E. Besser, J. M. Gay, L. K. Fox, M. A. Davis, R. N. Cobbold, A. C. B. Berge, and D. D. Hancock. 2009. The role of animal movement, including off-farm rearing of heifers, in the interherd transmission of multidrug-resistant Salmonella. J. Dairy Sci. 92:4229-4238.

Anderson, R. J., J. K. House, B. P. Smith, H. Kinde, R. L. Walker, B. J. Vande Steeg, and R. E. Breitmeyer. 2001. Epidemiologic and biological characteristics of salmonellosis in three dairy herds. J. Am. Vet. Med. Assoc. 219:310-322.

Anderson, R. J., R. L. Walker, D. W. Hird, and P. C. Blanchard. 1997. Case-control study of an outbreak of clinical disease attributable to Salmonella Menhaden infection in eight dairy herds. J. Am. Vet. Med. Assoc. 210:528-530.

Bauer, A. W., W. M. Kirby, J. C. Sherris, and M. Turck. 1966. Antibiotic susceptibility testing by a standardized single disk method. Am. J. Clin. Pathol. 45:493-496.

Berge, A. C., J. M. Adaska, and W. M. Sischo. 2004. Use of antibiotic susceptibility patterns and pulsed-field gel electrophoresis to compare historic and contemporary isolates of multi-drug-resistant Salmonella enterica subsp. enterica serovar Newport. Appl. Environ. Microbiol. 70:318-323.

Berge, A. C., D. A. Moore, and W. M. Sischo. 2006. Prevalence and antimicrobial resistance patterns of Salmonella enterica in preweaned calves from dairies and calf ranches. Am. J. Vet. Res. $67: 1580-1588$

Bezanson, G. S., R. Khakhria, and E. Bollegraaf. 1983. Nosocomial outbreak caused by antibiotic-resistant strain of Salmonella Typhimurium acquired from dairy cattle. Can. Med. Assoc. J. 128:426-427.

Blodgett, R. 2006. Most probable number from serial dilutions. In FDA's bacteriological analytical manual. Appendix 2: http://www. cfsan.fda.gov/ebam/bam-a2.html.

Butaye, P., G. B. Michael, S. Schwarz, T. J. Barrett, A. Brisabois, and D. G. White. 2006. The clonal spread of multidrug-resistant nontyphi Salmonella serotypes. Microbes Infect. 8:1891-1897.

CDC. 2005. Salmonella surveillance: Annual summary, 2005. US Department of Health and Human Services, Atlanta, GA.

Chambers, P. G., and R. J. Lysons. 1979. The inhibitory effect of bovine rumen fluid on Salmonella Typhimurium. Res. Vet. Sci. 26:273-276.

Cobbold, R. N., D. H. Rice, M. A. Davis, T. E. Besser, and D. D. Hancock. 2006. Long-term persistence of multi-drug-resistant
Salmonella enterica serovar Newport in two dairy herds. J. Am. Vet. Med. Assoc. 228:585-591.

Davis, M. A., T. E. Besser, K. Eckmann, K. Macdonald, D. Green, D. D. Hancock, K. N. Baker, L. D. Warnick, Y. Soyer, M. Wiedmann, and D. R. Call. 2007a. Multidrug-resistant Salmonella Typhimurium, Pacific Northwest, United States. Emerg. Infect. Dis. $13: 1583-1586$

Davis, M. A., D. D. Hancock, and T. E. Besser. 2002. Multiresistant clones of Salmonella enterica: The importance of dissemination. J. Lab. Clin. Med. 140:135-141.

Davis, M. A., D. D. Hancock, T. E. Besser, J. B. Daniels, K. N. Baker, and D. R. Call. 2007b. Antimicrobial resistance in Salmonella enterica serovar Dublin isolates from beef and dairy sources. Vet. Microbiol. 119:221-230.

Davis, M. A., D. D. Hancock, D. H. Rice, D. R. Call, R. DiGiacomo, M. Samadpour, and T. E. Besser. 2003. Feedstuffs as a vehicle of cattle exposure to Escherichia coli O157:H7 and Salmonella enterica. Vet. Microbiol. 95:199-210.

Davison, H. C., A. R. Sayers, R. P. Smith, S. J. Pascoe, R. H. Davies, J. P. Weaver, and S. J. Evans. 2006. Risk factors associated with the Salmonella status of dairy farms in England and Wales. Vet. Rec. 159:871-880.

Davison, H. C., R. P. Smith, S. J. Pascoe, A. R. Sayers, R. H. Davies, J. P. Weaver, S. A. Kidd, R. W. Dalziel, and S. J. Evans. 2005. Prevalence, incidence and geographical distribution of serovars of Salmonella on dairy farms in England and Wales. Vet. Rec. 157:703-711.

Edrington, T. S., T. R. Callaway, R. C. Anderson, and D. J. Nisbet. 2008. Prevalence of multidrug-resistant Salmonella on commercial dairies utilizing a single heifer raising facility. J. Food Prot. 71:27-34.

Evans, S. J. 1996. A case control study of multiple resistant Salmonella Typhimurium DT 104 infection of cattle in Great Britain. Cattle Pract. 4:259-266.

FAO/WHO. 2007. Animal Feed Impact on Food Safety. Report of the FAO/WHO Expert Meeting. FAO, Rome, Italy.

Fey, P. D., T. J. Safranek, M. E. Rupp, E. F. Dunne, E. Ribot, P. C. Iwen, P. A. Bradford, F. J. Angulo, and S. H. Hinrichs. 2000. Ceftriaxone-resistant Salmonella infection acquired by a child from cattle. N. Engl. J. Med. 342:1242-1249.

Hancock, D. 1996. Strategic laboratory use: How to use hypothesisbased laboratory testing. Proc. 28th Annu. Mtg. Am. Assoc. Bovine Pract. 28:118-125.

Hancock, D., T. E. Besser, J. M. Gay, D. Rice, M. Davis, and C. Gay. 2000. The global epidemiology of multiresistant Salmonella enterica serovar Typhimurium DT104. Pages 217-243 in Emerging Diseases of Animals. C. Brown and C. A. Bolin, ed. ASM Press, Washington, DC.

Jordan, D. 2005. Simulating the sensitivity of pooled-sample herd tests for fecal Salmonella in cattle. Prev. Vet. Med. 70:59-73.

Langvad, B., M. N. Skov, E. Rattenborg, J. E. Olsen, and D. L. Baggesen. 2006. Transmission routes of Salmonella Typhimurium DT104 between 14 cattle and pig herds in Denmark demonstrated by molecular fingerprinting. J. Appl. Microbiol. 101:883-890.

Lindqvist, N., S. Heinikainen, A. M. Toivonen, and S. Pelkonen. 1999. Discrimination between endemic and feedborne Salmonella Infantis infection in cattle by molecular typing. Epidemiol. Infect. 122:497-504.

Mattila, T., A. J. Frost, and D. O'Boyle. 1988. The growth of Salmonella in rumen fluid from cattle at slaughter. Epidemiol. Infect. 101:337-345.

McPherson, M. E., J. E. Fielding, B. Telfer, N. Stephens, B. G. Combs, B. A. Rice, G. J. Fitzsimmons, and J. E. Gregory. 2006. A multijurisdiction outbreak of Salmonella Typhimurium phage type 135 associated with purchasing chicken meat from a supermarket chain. Commun. Dis. Intell. 30:449-455.

Mead, P. S., L. Slutsker, V. Dietz, L. F. McCaig, J. S. Bresee, C. Shapiro, P. M. Griffin, and R. V. Tauxe. 1999. Food-related illness and death in the United States. Emerg. Infect. Dis. 5:607-625.

NCCLS. 2003a. National Committee for Clinical Laboratory Standards, Approved Standard M2-A8. Performance Standards 
for Antimicrobial Disk Susceptibility Tests. 8th ed. CLSI (formerly NCCLS), Wayne, PA.

NCCLS. 2003b. National Committee for Clinical Laboratory Standards, M100-S13(M2). Disk Diffusion Supplemental Tables. CLSI (formerly NCCLS), Wayne, PA.

Nielsen, L. R., B. van den Borne, and G. van Schaik. 2007a. Salmonella Dublin infection in young dairy calves: Transmission parameters estimated from field data and an SIR-model. Prev. Vet. Med. 79:46-58.

Nielsen, L. R., L. D. Warnick, and M. Greiner. 2007b. Risk factors for changing test classification in the Danish surveillance program for Salmonella in dairy herds. J. Dairy Sci. 90:2815-2825.

Popoff, M. Y. 2001. Antigenic formulas of the Salmonella serovars. WHO collaborating centre for reference and research on Salmonella. WHO, Paris, France.

Ribot, E. M., R. K. Wierzba, F. J. Angulo, and T. J. Barrett. 2002. Salmonella enterica serotype Typhimurium DT104 isolated from humans, United States, 1985, 1990, and 1995. Emerg. Infect. Dis. 8:387-391.

Richardson, A. 1975. Outbreaks of bovine salmonellosis caused by serotypes other than $S$. Dublin and $S$. Typhimurium. J. Hyg. (Lond.) 74:195-203.

Sanchez, S., C. L. Hofacre, M. D. Lee, J. J. Maurer, and M. P. Doyle. 2002. Animal sources of salmonellosis in humans. J. Am. Vet. Med. Assoc. 221:492-497.

Smith, B. P., F. Habasha, M. Reina-Guerra, and A. J. Hardy. 1979. Bovine salmonellosis: Experimental production and characterization of the disease in calves, using oral challenge with Salmonella Typhimurium. Am. J. Vet. Res. 40:1510-1513.

Spier, S. J., B. P. Smith, J. S. Cullor, H. J. Olander, L. D. Roden, and G. W. Dilling. 1991. Persistent experimental Salmonella Dublin intramammary infection in dairy cows. J. Vet. Intern. Med. $5: 341-350$.

Spika, J. S., S. Waterman, G. Hoo, M. St Louis, R. Pacer, S. James, M. Bissett, L. Mayer, J. Chiu, and B. Hall..1987. Chloramphenicolresistant Salmonella Newport traced through hamburger to dairy farms. A major persisting source of human salmonellosis in California. N. Engl. J. Med. 316:565-570.

van Schaik, G., Y. H. Schukken, M. Nielen, A. A. Dijkhuizen, H. W Barkema, and G. Benedictus. 2002. Probability of and risk factors for introduction of infectious diseases into Dutch SPF dairy farms: A cohort study. Prev. Vet. Med. 54:279-289.

Velge, P., A. Cloeckaert, and P. Barrow. 2005. Emergence of Salmonella epidemics: The problems related to Salmonella enterica serotype Enteritidis and multiple antibiotic resistance in other major serotypes. Vet. Res. 36:267-288.

Warnick, L. D., J. B. Kaneene, P. L. Ruegg, S. J. Wells, C. Fossler, L. Halbert, and A. Campbell. 2003. Evaluation of herd sampling for Salmonella isolation on midwest and northeast US dairy farms. Prev. Vet. Med. 60:195-206.

Williams, B. M. 1975. Environmental considerations in salmonellosis. Vet. Rec. 96:318-321.

Wray, C., and W. J. Sojka. 1977. Reviews of the progress of dairy science: Bovine salmonellosis. J. Dairy Res. 44:383-425.

Wray, C., N. Todd, I. McLaren, Y. Beedell, and B. Rowe. 1990. The epidemiology of Salmonella infection of calves: The role of dealers. Epidemiol. Infect. 105:295-305.

Zansky, S., B. Wallace, D. Schoonmaker-Bopp, P. Smith, F. Ramsey, J. Painter, A. Gupta, P. Kalluri, and S. Noviello. 2002. From the Centers for Disease Control and Prevention. Outbreak of multidrug resistant Salmonella Newport-United States, January-April 2002. JAMA 288:951-953. 